\title{
Magnetoresistance of $\mathrm{Si} / \mathrm{Nb} / \mathrm{Si}$ Trilayers
}

\author{
I. Zaytseva ${ }^{a}$, M.Z. Cieplak $^{a}$, A. Abal'oshev ${ }^{a}$, P. Dluzewski ${ }^{a}$, G. Grabecki $^{a}$, \\ W. PlesiewiCZ ${ }^{a}$, L.Y. ZHU ${ }^{b}$ AND C.L. Chien ${ }^{b}$ \\ ${ }^{a}$ Institute of Physics, Polish Academy of Sciences, al. Lotników 32/46, 02-668 Warsaw, Poland \\ ${ }^{b}$ Department of Physics and Astronomy, The Johns Hopkins University, Baltimore, MD 21218, USA
}

We study the superconductor-insulator transition in $\mathrm{Si} / \mathrm{Nb} / \mathrm{Si}$ trilayers, in which the thickness of $\mathrm{Si}$ is fixed at $10 \mathrm{~nm}$, and the nominal thickness of $\mathrm{Nb}$ changes in the range between $d=20 \mathrm{~nm}$ down to $d=0.3 \mathrm{~nm}$. The transmission electron microscopy indicates the formation of the mixed Nb-Si layer for small $d$. Both the thickness-induced, and the magnetic-field induced superconductor-insulator transition is observed. The crossing point of the isotherms at the critical field $B_{\mathrm{c}}$ decreases with decreasing $d$, and it is $T$-independent at temperatures below $300 \mathrm{mK}$. At larger fields the weak peak in magnetoresistance appears in some of the films.

PACS numbers: 74.62.-c, 74.25.F-, 74.25.Ha, 74.62.En

\section{Introduction}

The reduction of the thickness of superconducting films, $d$, increases the disorder and leads eventually to the thickness-induced superconductor-insulator transition $(d$-SIT). The transition to the insulating phase may be also induced by the presence of external magnetic field $B$ (B-SIT). This evolution remains one of the most important and perplexing problems in condensed matter physics. Many experimental and theoretical studies have been devoted to the subject, but the nature of the SIT is still under debate. It has been suggested recently that various materials may be divided into two groups depending on the behavior of the resistance in the vicinity of the SIT [1]. The first group are the strongly disordered systems with small carrier concentration, $n$, such as $\mathrm{InO}_{x}$ or $\mathrm{TiN}$, in which the resistance rises exponentially with lowering of the temperature, $T$, and huge magnetoresistance peak is observed on the insulating side of the $B$-SIT $[2,3]$. The second group includes materials with large $n$ and small disorder, such as, for example, MoGe, $\mathrm{Bi}, \mathrm{PbBi}$ or $\mathrm{Nb}_{1-x} \mathrm{Si}_{x}$. In these systems the resistance grows only logarithmically with the decreasing temperature and the magnetoresistance increase in the $B$-SIT is rather modest $[4,5]$. Recent theories predict that different $B$-SIT appears in these two groups. While the uniform suppression of the superconducting order parameter with increasing $B$ is believed to occur in weakly disordered systems with large $n$, the presence of superconducting islands immersed in the insulating matrix is expected in the strongly disordered materials with small $n$ [6]. To verify this prediction it would be interesting to study systems which could be tuned between these two extreme limits, which may be presumably achieved by tuning either the $n$, or the disorder, or both. However, so far there were no reports on this type of tuning. In the present work we describe the attempt to fabricate such a system.

\section{Experimental details and results}

We grow and study a set of $\mathrm{Si} / \mathrm{Nb} / \mathrm{Si}$ trilayers, in which the thickness of $\mathrm{Si}$ is fixed at $10 \mathrm{~nm}$, and the nominal $\mathrm{Nb}$ thickness $d$ is decreased from $20 \mathrm{~nm}$ down to $0.1 \mathrm{~nm}$. The samples are grown on glass substrates by sputtering in the high-vacuum chamber at room temperature. Figure 1 shows two images obtained by cross-sectional transmission electron microscopy (TEM), for $d$ equal to $10 \mathrm{~nm}$ and $0.3 \mathrm{~nm}$. It is seen that the interfaces between Si and $\mathrm{Nb}$ layers are very sharp. The nominal thickness of the thicker sample agrees well with the measured thickness. However, as $d$ is reduced, the observed thickness of the $\mathrm{Nb}$ film becomes progressively thicker than the nominal one, reaching about 5 times more in the case of nominal $d=0.3 \mathrm{~nm}$ film. This result suggests the diffusion of the light $\mathrm{Si}$ atoms into niobium film, and formation of the thicker mixed $\mathrm{Nb}-\mathrm{Si}$ layer. On the other hand, larger $\mathrm{Nb}$ atoms do not diffuse into silicon layer, so that the interfaces of the mixed alloy layer are well defined.

The resistance per square, $R_{\mathrm{sq}}$, is measured on a lithographically patterned resistance bridge using a standard four-probe method, by dc measurements at high $T(>2 \mathrm{~K})$, and by the ac measurements in the millikelvin $T$-range. Figure 2 shows the $R_{\mathrm{sq}}(T)$-dependence for a set of trilayers with different $d$. The superconducting transition temperature, $T_{\mathrm{c}}$, is reduced as $d$ decreases and the $R_{\mathrm{sq}}$ grows. The $R_{\mathrm{sq}}(T)$ undergoes gradual evolution, from the samples in which $\delta R_{\mathrm{sq}} / \delta T>0$ at finite temperatures ("superconducting" phase) to samples in which $\delta R_{\mathrm{sq}} / \delta T<0$ ("insulating" phase). This may be identified as the $d$-SIT, which occurs at $d$ about $0.3 \mathrm{~nm}$. The transition is most likely induced by both the increase of disorder and the decrease of $n$. The decrease of the carrier concentration may be expected in case of $\mathrm{Nb}-\mathrm{Si}$ alloy, because the mixed compound $\mathrm{Nb}_{1-x} \mathrm{Si}_{x}$ is known to undergo a metal-insulator transition at $x=0.13$ as a result of the decrease of $n$ [7]. 


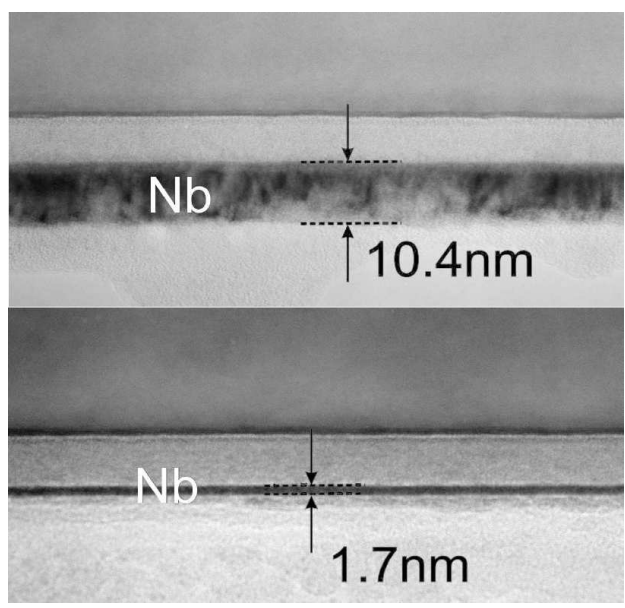

Fig. 1. TEM images for $\mathrm{Si} / \mathrm{Nb} / \mathrm{Si}$ trilayers with the nominal thickness of niobium, $d$, equal to $10 \mathrm{~nm}$ (top) and $0.3 \mathrm{~nm}$ (bottom). The layers in both images, from top to bottom, are: substrate, amorphous $\mathrm{Si}$, Nb (with the measured thickness indicated in the figure), and $\mathrm{Si}$ covered with glue.

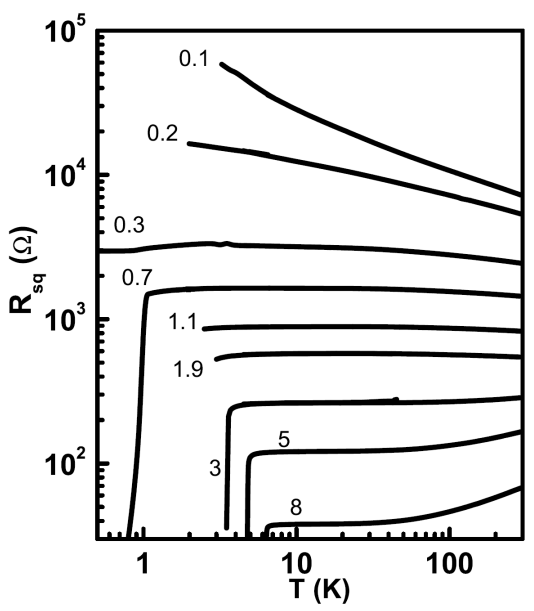

Fig. 2. The dependence of $R_{\mathrm{sq}}$ on $T$ for a set of trilayers. The labels next to the data indicate the nominal thickness of $\mathrm{Nb}$ (in $\mathrm{nm}$ ), from 0.1 (top) to 8 (bottom).

Despite the expected decrease of $n$, the thinnest films show relatively weak (logarithmic) increase of the resistance with the lowering of temperature. This remains in marked contrast to the activated behavior of the resistance, which is observed in films of $\mathrm{InO}_{x}$ [2], $\mathrm{TiN}$ [3], or $\mathrm{Bi}[8]$. Therefore, it seems that the $\mathrm{Si} / \mathrm{Nb} / \mathrm{Si}$ trilayers belong to a second group of films described in the introduction, i.e. to a group of weakly disordered materials.

The dependences of $R_{\mathrm{sq}}$ on $B$, applied perpendicular to the sample plane, has been measured, and two examples, for samples with $d=0.7 \mathrm{~nm}$ and $d=0.4 \mathrm{~nm}$, are shown in Figs. 3 and 4, respectively. The main part of Fig. 3 shows the resistance isotherms, measured as function of $B$ at fixed temperatures between $80 \mathrm{mK}$ and $300 \mathrm{mK}$. The isotherms in Fig. 4 are measured between $60 \mathrm{mK}$ and
$500 \mathrm{mK}$. The sample with $d=0.7 \mathrm{~nm}$ is superconducting at $B=0$ at the lowest temperatures, while the sample with $d=0.4 \mathrm{~nm}$ does not show a complete transition to superconducting state. However, a lowering of resistance is seen, indicating the presence of superconducting fluctuations. Defining as $T_{\mathrm{c}}$ the temperature at which $R / R_{\mathrm{N}}=0.5$ for superconducting samples $\left(R_{\mathrm{N}}\right.$ is the normal-state resistance at $T=10 \mathrm{~K})$ we extract $T_{\mathrm{c}}(B)$, from which we estimate the coherence length, $\xi(0)$. $\xi(0)$ is found to decrease from $22 \mathrm{~nm}$ to $14 \mathrm{~nm}$ when $d$ is reduced from 10 to $0.7 \mathrm{~nm}$, indicating that the mean free path decreases by a factor of about 2.5, consistent with the approach to the SIT.

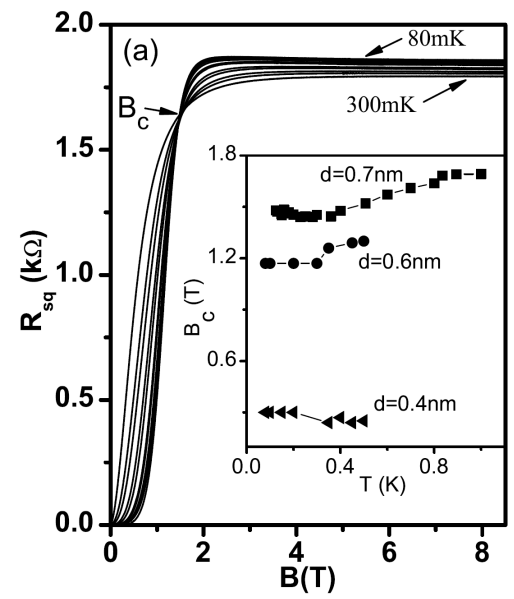

Fig. 3. The isotherms of $R_{\mathrm{sq}}(B)$ measured for sample with $d=0.7 \mathrm{~nm}$ at temperatures between $80 \mathrm{mK}$ (top curve) and $300 \mathrm{mK}$ (bottom curve). The arrow indicates crossing point. Inset: the dependences on the temperature of the $B_{\mathrm{c}}$ for films with $d=0.7 \mathrm{~nm}, d=0.6 \mathrm{~nm}$, $d=0.4 \mathrm{~nm}$.

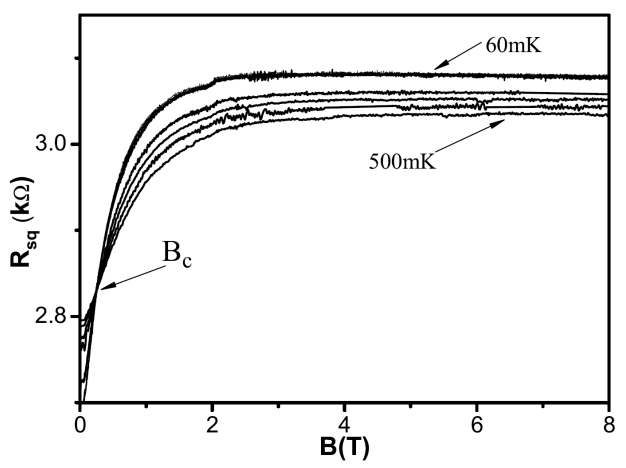

Fig. 4. The isotherms of $R_{\mathrm{sq}}(B)$ measured for sample with $d=0.4 \mathrm{~nm}$ at temperatures between $60 \mathrm{mK}$ (top curve) and $500 \mathrm{mK}$ (bottom curve). The arrow indicates crossing point.

In both figures we observe a crossing point of the resistance isotherms at some critical field $B_{\mathrm{c}}$ and critical resistance $R_{\mathrm{c}}$. The $B_{\mathrm{c}}$ may be identified as the magnetic 
field at which the $B$-SIT takes place $[2,3,8]$. This is because at $B<B_{\mathrm{c}}$ we have $\delta R_{\mathrm{sq}} / \delta T>0$ (characteristic for superconducting phase), and for $B>B_{\mathrm{c}}$ we have $\delta R_{\mathrm{sq}} / \delta T<0$ (insulating behavior). It follows that the signature of the $B$-SIT would be a $T$-independent crossing point, at which $\delta R_{\mathrm{sq}} / \delta T=0$.

The presence of the crossing point and the change of sign of $\delta R / \delta T$ is predicted by the "dirty boson model" of the SIT [9]. In accord with the model the coherence of the superconducting state is destroyed by quantum fluctuations of the order parameter's phase, and the system amounts to interacting bosons in the presence of disorder. The superconducting and insulating phases are then dual to one another: the superconducting phase consists of localized vortices and condensed Cooper pairs, whereas the insulating phase is characterized by condensed vortices and localized Cooper pairs.

In order to verify the $T$-independence of the crossing point, we plot in the inset to Fig. 3 the $T$-dependence of the $B_{\mathrm{c}}$, defined as the field at which two consecutive isotherms cross. The $B_{\mathrm{c}}$ is measured in the $T$ -range between $80 \mathrm{mK}$ and $1 \mathrm{~K}$ for $d=0.7 \mathrm{~nm}$ sample, and between $60 \mathrm{mK}$ and $500 \mathrm{mK}$ for $d=0.6 \mathrm{~nm}$ and $d=0.4 \mathrm{~nm}$. The $B_{\mathrm{c}}$ is seen approximately constant for $T \lesssim 0.3 \mathrm{~K}$, while for higher $T$ it starts to change. This indicates that the $T$-independence of the crossing point breaks down at higher temperatures. In this respect, our results for trilayers seem to be comparable to these reported for $\mathrm{Nb}_{1-x} \mathrm{Si}_{x}$ films [5], although the breakdown occurs at somewhat lower $T$ in trilayers. This may be related to smaller $n$, and to larger disorder in trilayers in comparison to relatively thick $\mathrm{Nb}_{1-x} \mathrm{Si}_{x}$ films described in Ref. [5].

An interesting feature is the weak magnetoresistance peak, which is seen at lowest temperatures at $B>B_{\mathrm{c}}$ in the sample with $d=0.7 \mathrm{~nm}$. On the other hand, the peak appears to be absent in the sample with $d=0.4 \mathrm{~nm}$. The peak resembles somewhat the magnetoresistance peak observed in strongly disordered films of $\mathrm{InO}_{x}$ and $\mathrm{TiN}$ $[2,3]$, except that in these materials the peak is huge, and it seems to grow with the growing disorder in the films. The microwave experiments indicate the presence of vortices in the peak region in $\mathrm{InO}_{x}$ [10], while STM experiment on TiN suggests that superconductivity in these samples is confined to superconducting islands immersed in the insulating matrix [11]. In the group of weakly disordered materials, mentioned in the introduction, the magnetoresistance peak is not described, probably because it is either very weak or altogether absent. It seems that the observation of this feature in some of our samples indicates that we have succeeded in growing samples with some intermediate behavior between weakly and highly disordered materials. The presence or absence of the peak may be related to a specific degree of disorder which builds randomly into our very thin samples.

The origin of the magnetoresistance peak is still under debate. The "dirty boson model" predicts the existence of the insulating state with localized Cooper pairs at $B>B_{\mathrm{c}}$. However, it is unclear how to interpret in this context the decrease of the resistance at higher $B$. A different proposal assumes a large role of superconducting fluctuations [12], which may stimulate the electron localization, and lead to the appearance of magnetoresistance peak [1]. Further studies of the trilayer system (including the effect of parallel $B$ ) should help to elucidate the origin of this feature.

In conclusion, we have grown the $\mathrm{Si} / \mathrm{Nb} / \mathrm{Si}$ trilayers in which the decrease of the nominal $\mathrm{Nb}$ thickness to very small value results in the formation of $\mathrm{Nb}-\mathrm{Si}$ mixed layer. We have observed the $d$-SIT and the $B$-SIT in this system. The transitions are most likely caused by both the disorder, and by the decrease of the carrier concentration in comparison with the bulk $\mathrm{Nb}$. The presence of magnetoresistance peak above the $B_{\mathrm{c}}$ indicates the intermediate range of disorder in the trilayers.

\section{Acknowledgments}

This work was supported by Polish MNiSW grant N202 058 32/1202, and by NSF grant DMR05-20491.

\section{References}

[1] V.F. Gantmakher, Physica C 404, 176 (2004).

[2] M. Steiner, A. Kapitulnik, Physica C 422, 16 (2005).

[3] T.I. Baturina, C. Strunk, M.R. Baklanov, A. Satta, Phys. Rev. Lett. 98, 127003 (2007).

[4] A. Yazdani, A. Kapitulnik, Phys. Rev. Lett. 74, 3037 (1995).

[5] H. Aubin, C.A. Marrache-Kikuchi, A. Pourret, K. Behnia, L. Berge, L. Dumoulin, J. Lesueur, Phys. Rev. B 73, 094521 (2006).

[6] Y. Dubi, Y. Meir, Y. Avishai, Nature 449, 876 (2007).

[7] G. Hertel, D.J. Bishop, E.G. Spencer, J.M. Rowell, R.C. Dynes, Phys. Rev. Lett. 50, 743 (1983).

[8] N. Markovic, C. Christiansen, A.M. Goldman, Phys. Rev. Lett. 81, 5217 (1998).

[9] M.P.A. Fisher, Phys. Rev. Lett. 65, 923 (1990).

[10] R. Crane, N.P. Armitage, A. Johansson, G. Sambandamurthy, D. Shahar, G. Gruner, Phys. Rev. B 75, 184530 (2007).

[11] B. Sacepe, C. Chapelier, T.I. Baturina, V.M. Vinokur, M.R. Baklanov, M. Sanquer, Phys. Rev. Lett. 101, 157006 (2008).

[12] V.M. Galitski, A.I. Larkin, Phys. Rev. B 63, 174506 (2001). 